\title{
A Simple laboratory Model for investigating the Dynamic Instability of a Nearly Two-dimentional Jet in a Rotating Fluid
}

\author{
By Toshio Yamagata* and Ryuji Kimura** \\ (Manuscript received 10 March 1973, in revised form 15 October 1973)
}

\begin{abstract}
An experimental study of the dynamic instability in a rotating system is presented. The basic jet is made by rubbing the free surface of water in rigid rotation with a solid plate. The structure of the basic jet is studied by a linear theory on the vertical free shear layers. The beta-effect is simulated by variation of the fluid depth.

When the Rossby number exceeds a certain critical value, the zonal jet becomes unstable and a disturbance with a specific wave number appears. By comparing the observed wave numbers with relevant linear theories, the following results are obtained.

(i) The observed marginal state better fits the theory in which the jet is stabilized by the beta-effect than the theory in which the jet is stabilized by the internal viscosity.

(ii) The observed results show that the westerly jet is more stable than the easterly jet.

(iii) The observed wave number rapidly decreases from the linear mode, when the Rossby number increases from the critical value.
\end{abstract}

\section{Introduction}

This paper presents a simple laboratory model for a study of dynamic instability in a rotating fluid. Although we have many linear theories for an inviscid two-dimensional jet (for example, Kuo, 1949; Lipps, 1962, 1965; Yanai and Nitta, 1968; Yamasaki and Wada, 1972), our knowledge about the properties of the finite-amplitude disturbance in the dissipative system is not enough at the present stage. In general we have the following images for the evolution of the disturbance: When the basic state becomes unstable, a disturbance with a specific wave number (predicted by the linear theories) appears and grows exponentially at first. But soon the growth is suppressed by non-linear effects and the system falls into a new equilibrium where the finite-amplitude disturbance is superposed on the new basic state (which is defined through the averaging process of the new state). The structure of the disturbance in the final state may be classified into the following

* Geophysical Institute, University of Tokyo, Tokyo (Currently the Ocean Research Institute, University of Tokyo, Tokyo.)

** Ocean Research Institute, University of Tokyo, Tokyo. three cases; i) the linear preferred mode continues to exist, ii) the disturbances with various wave numbers are generated through the non-linear process and the system becomes turbulent, iii) the disturbance has structure that is different from the linear mode. It is known that the dynamic instability in the non-rotating system has a strong tendency to take the case ii) (Sato, 1959). How about the dynamic instability in the rotating system? Since the dynamic instability in the rotating system is an important topic in geophysical sciences, it may be of interest to observe the behaviours of finite-amplitude disturbances realized in controlled laboratory experiments and compare the results with those obtained from the linear theories.

An essential point in laboratory experiments is how to construct a rotating fluid system in which a two-dimensional jet is maintained. It is found that a nearly two-dimensional jet whose velocity profile has inflection points is produced by making the following fluid system: The working fluid is a viscous, homogeneous and incompressible fluid in a cylindrical container (with radius $R$ and height $L$ ) which rotates about the vertical axis with an angular velocity $\Omega$. If all boundaries rotate with the same angular velocity, no motion 
occurs relative to the container in the steady state. We try to make a zonal flow by rubbing the fluid surface with a ring-shaped region (defined by $r_{1} \leq r \leq r_{2}<R$ ) which rotates with a slightly different speed from $\Omega$. The configuration is schematically shown in Fig. 1. Our fluid system

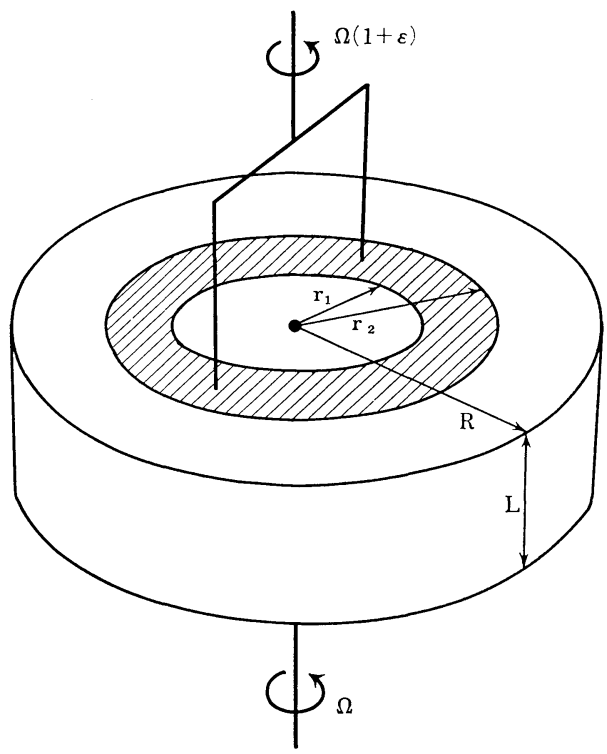

Fig. 1. Schematic representation of the fluid configuration.

may be regarded as a modification of the experiment performed by Hide and Titman (1967). The modified points are the velocity profile of the basic flow and the inclusion of the effect equivalent to the so-called beta-effect. These two modifications make it possible to compare the experimental results with those obtained by the linear theories for meteorological application.

In the next section the experimental arrangement of a laboratory model is described. The results of the laboratory experiment are described and discussed in Section 3. The structure of the basic flow is considered theoretically in the Appendix.

\section{A laboratory model}

\subsection{Experimental arrangement}

We tried to materialize the fluid system shown in Fig. 1 by making experimental apparatus as shown in Fig. 2. The working fluid is tap water in a cylindrical container with a plane bottom of inner radius $10.3 \mathrm{~cm}$. The container (made of acrylic resin) is installed on the turntable of a

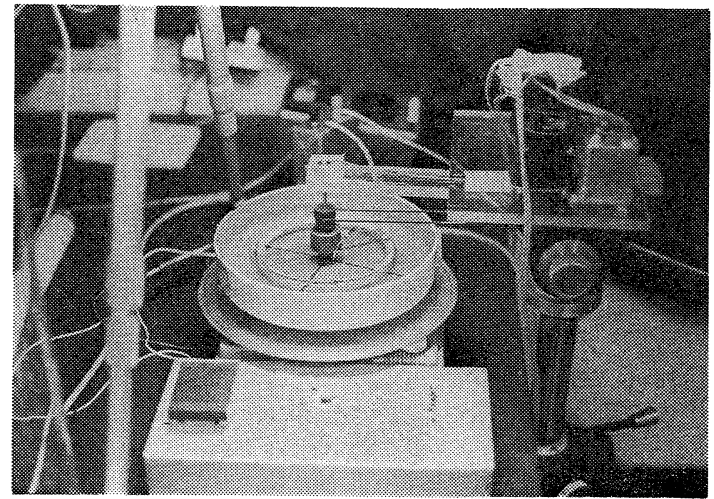

Fig. 2. Experimental apparatus.

stereo-player (Sony, TTS-2400, servo-controlled belt drive system), and the angular speed of the container is fixed at $33.3 \mathrm{RPM}(3.49 \mathrm{rad} / \mathrm{sec})$ or $45 \mathrm{RPM}(4.71 \mathrm{rad} / \mathrm{sec})$. The container is open to the ambient air, so that in equilibrium the depth of the water layer, $h$, may vary with the radial distance, $r$, from the rotating axis.

$h$ is given by

$$
h=z_{0}+\frac{\Omega_{b}^{2}}{2 g} r^{2}
$$

where $z_{0}$ is the depth at the rotating axis, $\Omega_{b}$ the angular velocity of the container and $g$ the acceleration due to gravity. In the following the depth of the fluid is represented by $z_{0}$ which varies from $0.46 \mathrm{~cm}$ to $2.20 \mathrm{~cm}$ in the present experiment. The basic zonal flow of a jet type is produced by a ring made of acrylic resin (inner radius $6.0 \mathrm{~cm}$ and outer radius $6.5 \mathrm{~cm}$ ) that contacts with the water surface. The ring is rotated about the vertical axis by a servo-motor (Japan Servo, ESA-5S) whose angular velocity, $\Omega_{r}$, can be controlled independently of $\Omega_{b}$. The experiment was performed in a temperaturecontrolled laboratory to minimize the effects of stratification.

\subsection{Experimental procedure and flow visualization}

Flows in the container described above are observed in the following three steps:

EXP 1. Observation of the rigid rotation.

Since the free surface of the fluid layer contacts with the resting atmosphere, the wind drag produces a flow relative to the basic rotating system (see 3.1.). The $\mathrm{pH}$-indicater method 
reported by Baker (1966) is used to measure the radial distribution of the zonal velocity caused by the wind drag. A small amount of thymol blue is contaminated in the working fluid. Two electrodes (a tungsten wire of 5 microns in diameter as a cathode and a platinum wire of 100 microns in diameter as an anode) are stretched at the bottom parallel to a radius of the container: Blue color produced around the cathode by electrolysis of water is used as a fluid marker in the Lagrangian sense.

EXP 2. Observation of the basic state.

Hydrogen-bubble method reported by Schraub et al. (1965) is used to measure the radial distribution of the zonal velocity caused by the driving of the ring. The configuration of the electrodes is the same as that in EXP 1 but small hydrogen bubbles produced by electrolysis of water are used as a fluid marker. The positions of the bubbles are photographed and the velocity is calculated by dividing the distances of the bubbles from the cathode by the time that elapses from the moments of bubble production to that of photographing. This time is also read out from the same photograph by measuring the angle swept by the electrode in this time lapse.

Two-dimensionality of the zonal flow is checked qualitatively by the hydrogen-bubble method. In this case a tungsten wire is stretched vertically near the outer edge of the ring and bubbles are produced successively by switching the voltage impressed between the electrodes.

EXP 3. Observation of disturbances due to dynamic instability.

The present apparatus is not feasible to observe the fine structures of the disturbances, so that we cannot go beyond measuring the wave number of the disturbances. The $\mathrm{pH}$-indicater method is used for this latter purpose.

A circular copper plate of radius $6.25 \mathrm{~cm}$ and thickness 10 microns which is painted for insulation except for the circular edge is placed on the bottom of the container and used as the cathode. If the zonal flow is stable, a blue ring is produced from the edge of the copper plate under the driving ring. When the zonal flow becomes unstable, the colored region is twisted and the wave number of the disturb- ance is known from polaroid photographs of the twisted ring pattern.

\section{3. Accuracy of the experiments}

According to the specifications the flutter of the turntable is less than $0.06 \%$. We could not detect the effect of irregular rotation in EXP 1. If the rotation axis of the turntable is not parallel to the direction of gravity, the oscillating flow is produced in the fluid layer. This can be detected by the colored water produced from the tungsten wire in EXP 1. The turntable was levelled so that this oscillating motion could not be detected by visual observation.

If the axis of the driving ring is not identical to that of the basic rotation, the flow opposite to the driving direction is produced near the ring. In our experiment we carefully adjusted these two axies so that this kind of back flow could hardly be detected (see Fig. 5).

Accuracy of the velocity measurement based on the electrolysis method depends on the flow situations. In EXP 1, a blue line produced around the cathode slowly rotates relative to the basic rotating system without showing appreciable deformation or diffusion due to the flow generated by wind drag as described in Section 3.1. The error contained in the flow velocity measured from the time required for the blue line to sweep $10^{\circ}$ is considered to be several percent. In EXP 2, however, the individual datum is considered to contain an error of more than $10 \%$. We can obtain a smooth velocity profile only by averaging many data.

\section{Experimental results and discussions}

\section{1. Rigid rotation}

In EXP 1 it was observed that in the steady state the main body of water layer in the rotating container without an upper lid was in almost rigid rotation whose angular speed was slightly smaller than that of the basic rotating system. This deviation of the angular velocity of the water from that of the container is considered to be due to wind drag working on the water surface. The deviation of the angular speed was measured for water layers of various depths. As shown in Fig. 3 the deviation of the angular speed, $\Delta \Omega$, decreases with $z_{0}$ linearly in the observational range and can be expressed by the following experimental formulas: 


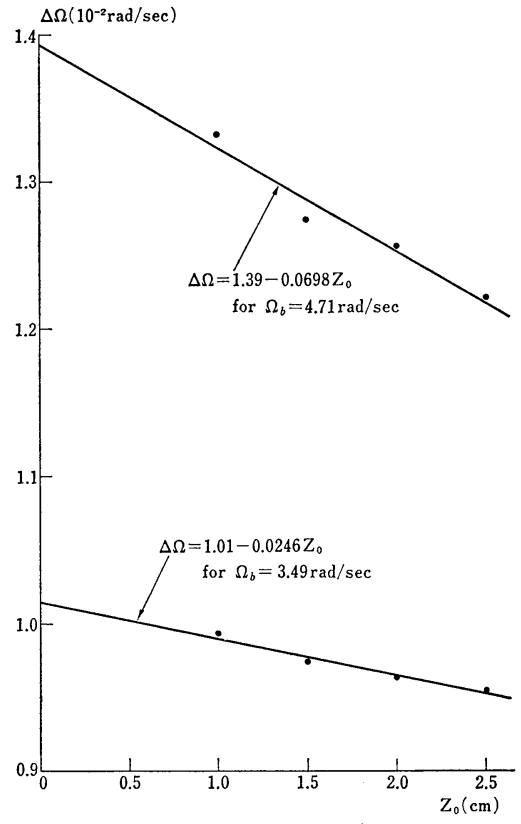

Fig. 3. Deviation of the angular speed from the solid rotation referred to $\Omega$. The deviation $\Delta \Omega$ is shown as a function of fluid depth $z_{0}$.

$$
\begin{aligned}
\Delta \Omega & =\left(1.01-0.0246 z_{0}\right) \\
& \times 10^{-2} \mathrm{rad} / \mathrm{sec} \text { for } \Omega_{b}=3.49 \mathrm{rad} / \mathrm{sec}, \\
\Delta \Omega & =\left(1.39-0.0698 z_{0}\right) \\
& \times 10^{-2} \mathrm{rad} / \mathrm{sec} \text { for } \Omega_{b}=4.71 \mathrm{rad} / \mathrm{sec} .
\end{aligned}
$$

Constants in the above formulas were obtained by the method of least mean square.

Since the flow pattern of a viscous fluid near a rotating disk is known (see p. 93 of Schlichting, 1968), it is possible to estimate the amount of deviation theoretically. Considering roughly that 1) $\Delta \Omega$ is half of the deviation at the free surface, 2) the vertical shear of the zonal velocity is constant and confined in the boundary layer whose thickness is $\left(\nu / \Omega_{b}\right)^{1 / 2}$ in the water layer, 3) the shape of the free surface is regarded as a disk, then we can write the relation which expresses the stress balance at the free surface of the water layer as

$$
\rho_{\nu} \frac{r \Delta \Omega}{\left(\frac{\nu}{\Omega_{b}}\right)^{1 / 2}}=\rho_{a} \nu_{a} \frac{d v_{a}}{d z} \mid \text { at the boundary }
$$

where $\rho_{a}, \nu_{a}, v_{a}$ are the density, the kinematic viscosity and the zonal velocity of the air respectively.
According to Schlichting (1968),

$$
\frac{d v_{a}}{d z} \mid \text { at the boundary }
$$

is given as $0.61 r \Omega_{b}^{3 / 2} \nu_{a}^{-1 / 2} . \quad \Delta \Omega$ can be calculated from (3) with this expression. The result is

$$
\begin{aligned}
& \Delta \Omega=0.99 \times 10^{-2} \mathrm{rad} / \mathrm{sec} \text { for } \Omega_{b}=3.49 \mathrm{rad} / \mathrm{sec} \text {, } \\
& \Delta \Omega=1.33 \times 10^{-2} \mathrm{rad} / \mathrm{sec} \text { for } \Omega_{b}=4.71 \mathrm{rad} / \mathrm{sec} \text {. }
\end{aligned}
$$

In spite of rough estimation the stress balance at the free surface well explains the observed deviation of the angular speed. Decrease of $\Delta \Omega$ with increase of $z_{0}$ may be explained by friction at the lateral wall of the fluid container.

\subsection{Basic flow driven by the ring}

a) Two-dimensionality

The thickness of the Ekman boundary layer in our experimental situation is estimated as

$$
\sqrt{\frac{\nu}{\Omega_{b}}}=0.05 \mathrm{~cm} \text { for } \Omega_{b}=3.49 \mathrm{rad} / \mathrm{sec}
$$

Therefore, the theoretical flow structure calculated in the Appendix is expected to be applicable almost to the whole fluid layer in the container. Two-dimensionality of the basic flow profile was checked qualitatively in two ways: 1) from the spread of a drop of ink that was contaminated in the water layer, 2) from the patterns of hydrogen-bubble lines that were produced from a tungsten wire stretched vertically in the water layer. Both ink and hydrogen-bubble patterns showed that the two-dimensionality of the flow was established fairly well. Fig. 4 is a photograph

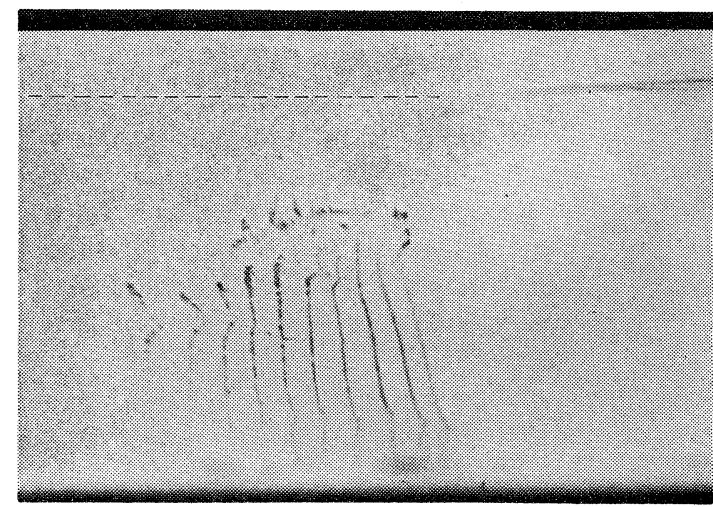

Fig. 4. Flow pattern in the vertical cross section near the ring visualized by hydrogen bubbles. The flow is directed from right to left. The broken line in the figure shows the upper boundary. 
of hydrogen-bubble lines taken through the lateral wall of the container. Hydrogen-bubble lines were produced near the outer edge of the ring and drifted downstream (from right to left in the figure). If there was a vertical shear in the flow, the line would tilt more and more according as it traveled downstream (cf. Fig. 4 in Kimura et al. (1971) which shows this case). Since the hydrogenbubble lines show no systematic tilt in Fig. 4, they may be regarded as evidence of the absence of vertical shear in the basic flow. Some irregularities seen in the figure were caused by the disturbances produced by the electrode and small filaments caught by the electrode.

b) Horizontal profiles.

Fig. 5 shows the observed velocity profiles of the zonal flow driven by the ring. The profiles
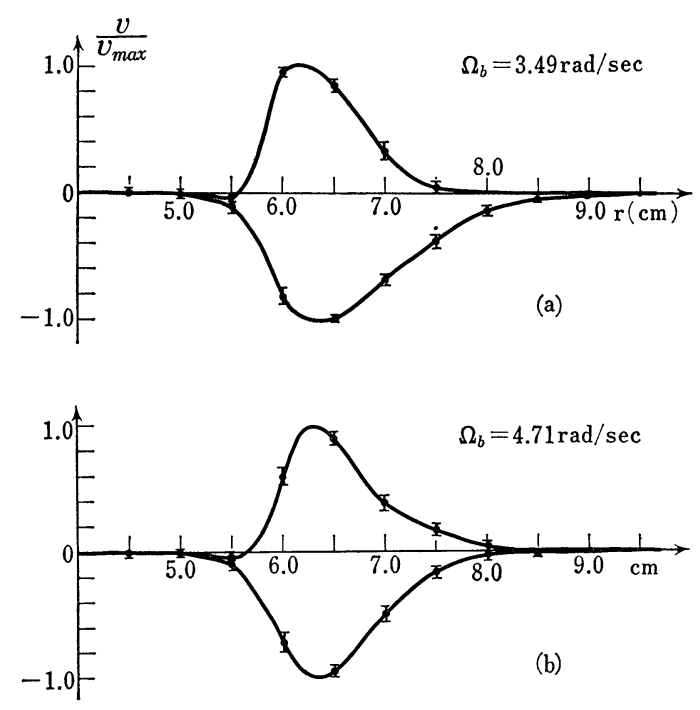

Fig. 5. Observed profiles of the zonal velocity normalized by the maximum value. They are classified by $\Omega_{b}$ and the direction of the flow relative to the basic rotating system (the positive value means that the flow is faster than the basic rotating system). A vertical segment at each point shows the standard deviation.

are normalized by the maximum velocity and classified by $\Omega_{b}$ and the direction of the flow relative to the basic rotating systems. The profiles for $\Omega_{b}=3.49 \mathrm{rad} / \mathrm{sec}$ were obtained by averaging the profiles for the fluid layer whose depth (at the rotating axis) is $0.70,1.20,1.70$ and $2.20 \mathrm{~cm}$. For each fluid layer the profiles were measured at
$R_{0}\left(\equiv \frac{0.25\left(\Omega_{r}-\Omega_{b}\right)}{\Omega_{b}}\right)=-0.045,-0.035,-0.016$, $0.013,0.027,0.041$ and 0.055 and averaged with respect to $R_{0}$. The profiles for $\Omega_{b}=4.71 \mathrm{rad} / \mathrm{sec}$ were obtained by the same procedure, but the fluid depth is $0.46,0.96,1.46$ and 1.96 and $R_{0}=-0.035,-0.024,-0.013,0.008,0.019,0.029$, and 0.040 . The standard deviations of the data are also shown in the figure. Although the flow profile ought to depend on the Ekman number, the accuracy of our velocity measurement was not enough to identify the dependence (Ekman number in the figure ranges from $5.5 \times 10^{-4}$ to $5.8 \times 10^{-3}$ ). In Fig. 6 the observed velocity profile obtained

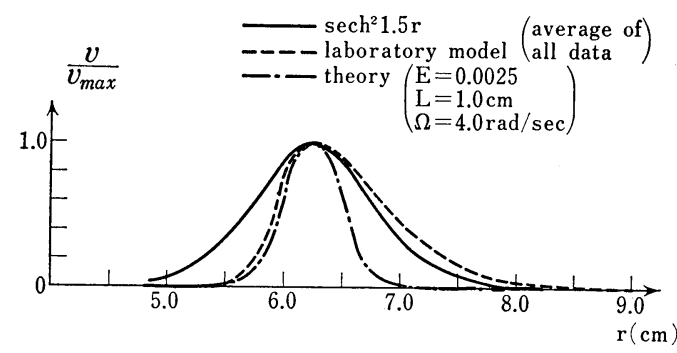

Fig. 6. Comparison of theory and experiment for zonal profile. The observed profile is obtained by averaging all data shown in Fig. 5. The theoretical profile is obtained for a typical combination of parameters. sech $^{2} 1.5 r$ is the profile used in the stability analysis in Section 3.3.

by averaging all data is compared with the theoretical profile calculated in the Appendix and $\operatorname{sech}^{2} b r$ which is often assumed as the typical jet profile in stability theories. Constant $b$ is rather arbitrarily determined as 1.5 in the figure. All profiles in Fig. 6 are normalized by the maximum velocity.

It is seen in these figures that 1) the observed profile is not symmetric with respect to the flow axis, 2) the width of the observed profile is broader than the predicted theoretical profile and 3) the width of the observed profile in the positive $y$-direction (corresponding to the westerly wind in the atmosphere) is smaller than that in the negative $y$-direction.

The feature 1) may be attributed to the curvature of the ring and to the variation of depth in the radial direction, which are neglected in the theory in the Appendix. The features 2) and 3) may be attributed to the fact that some data in 
Figs. 5 and 6 were obtained in situations where disturbances due to dynamic instability were superposed on the basic flow.

In such situations, the momentum given to the fluid by the ring is effectively transported in the radial direction, so that the mean flow width becomes broader. As described in 3.3. the flow in the negative $y$-direction is more unstable than the flow in the positive $y$-direction. This fact corresponds to the feature 3 ).

c) Amplitudes.

In Fig. 7., the ratios of the maximum zonal velocity to the velocity of the driving ring are

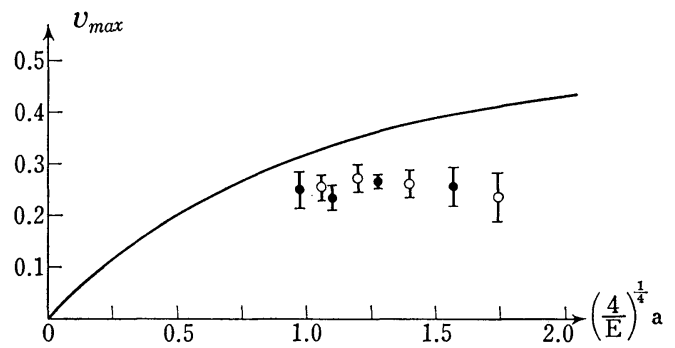

Fig. 7. Ratios of the maximum zonal velocity to the velocity of the driving ring. The solid curve is given by the formula (A-34). Circles and dots are experimental data for $\Omega_{b}=4.71 \mathrm{rad} / \mathrm{sec}$ and $\Omega_{b}=3.49 \mathrm{rad} / \mathrm{sec}$, respectively.

compared with the predicted value from (A-34). All data lie near $25 \%$ significantly below the theoretical values and appear to be independent of the Ekman number. This result may be partly explained by the effect of disturbances in the observational data, but we cannot find the reason why the observed ratios are independent of the Ekman number.

\section{3. Disturbances due to dynamic instability}

a) Phenomena Observed.

When $\Omega_{r}$ is near $\Omega_{b}$, the flow driven by the ring is axisymmetric but when $\delta \Omega\left(\equiv \Omega_{r}-\Omega_{b}\right)$ exceeds some critical value, a disturbance with a specific wave number appears in the zonal flow. It remains stable in finite amplitude, if the external parameters are fixed. This result makes a striking contrast with the results of Sato's experiment performed in the non-rotating system (Sato, 1959), in which the disturbances grow exponentially at first and then develop into turbulences. This fact will provide some interesting information for consid- ering the generation of turbulence in a rotating system.
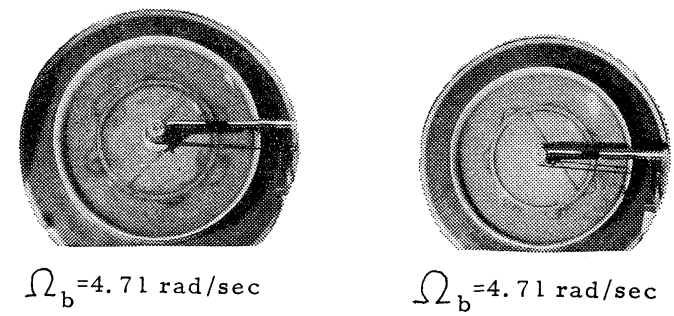

$\Omega_{\mathrm{b}}=4.71 \mathrm{rad} / \mathrm{sec}$

$\Omega_{\mathrm{r}}=3.27 \mathrm{rad} / \mathrm{sec}$

$\Omega_{\mathrm{r}}=3.67 \mathrm{rad} / \mathrm{sec}$

$Z_{0}=0.96 \mathrm{~cm}$

$Z_{\mathrm{o}}=0.46 \mathrm{~cm}$

$k=6$

$\mathrm{k}=7$
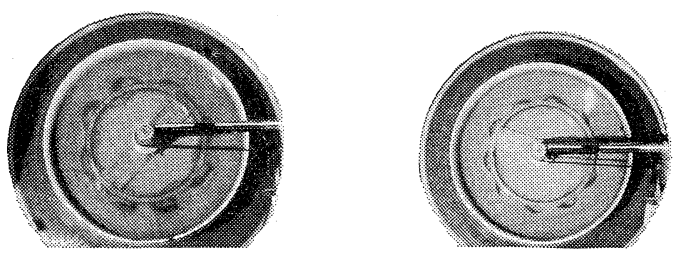

$\Omega_{\mathrm{b}}=4.71 \mathrm{rad} / \mathrm{sec}$

$\Omega_{\mathrm{b}}=4.7 \mathrm{rad} / \mathrm{sec}$

$\Omega_{\mathrm{r}}=3.67 \mathrm{rad} / \mathrm{sec}$

$\Omega_{\mathrm{r}}=4.01 \mathrm{rad} / \mathrm{sec}$

$Z_{0}=0.96 \mathrm{~cm}$

$\mathrm{k}=8$

$Z_{\mathrm{o}}=0.46 \mathrm{~cm}$

$\mathrm{k}=10$

Fig. 8. Patterns of the disturbances visualized by $\mathrm{pH}$-indicator method.

Fig. 8 shows examples of the disturbance visualized by the pH-indicater method. The observed wave number ranged from 2 to 11 according to various combinations of $\delta \Omega$ and $z_{0}$. The observed data for $\Omega_{b}=4.71 \mathrm{rad} / \mathrm{sec}$ are summarized in Fig 9 where the Rossby number defined by $R_{0} \equiv \frac{0.25 \delta \Omega}{\Omega_{b}}$ is used instead of $\frac{\delta \Omega}{\Omega_{b}}$. The quantity $0.25 \delta \Omega$ expresses approximately the maximum angular speed of the zonal flow. Notice the following features in Fig. 9: 1) The observed wave number at a fixed $R_{0}$ decreases as the fluid depth $z_{0}$ increases. 2) The critical Rossby number for instability is smaller in the negative Rossby number region (easterly region) than in the positive Rossby number region (westerly region).

In some combinations of the external parameters we observed a time-dependent flow pattern in which the wave number increased and decreased 


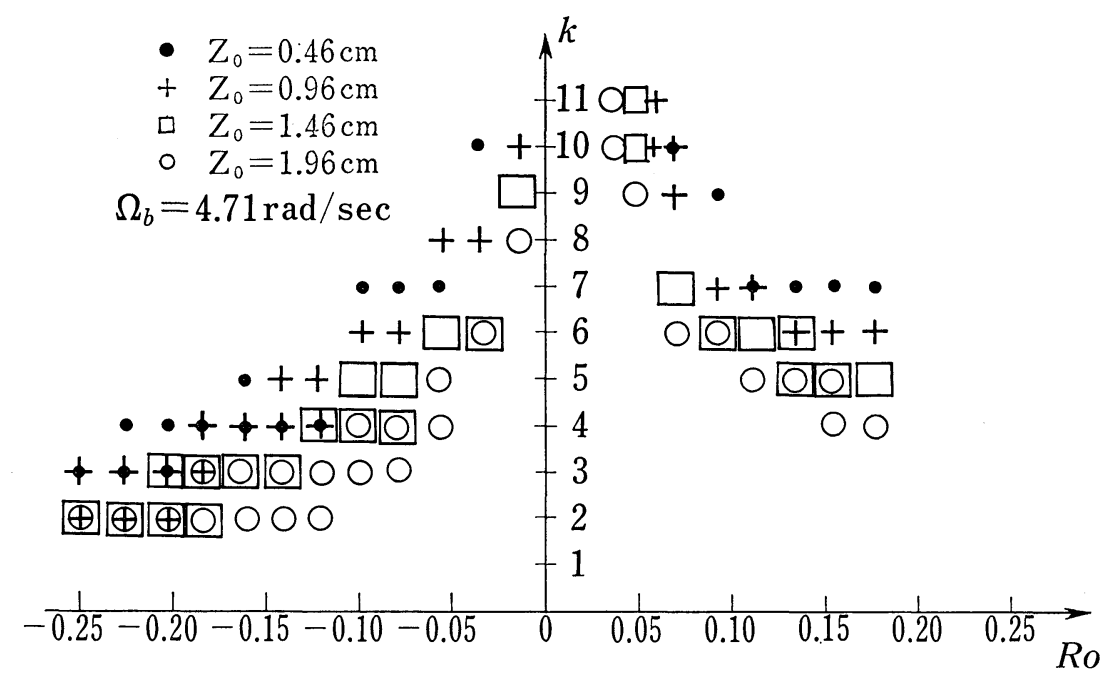

Fig. 9. Observed wave numbers plotted as a function of the Rossby number $\left(R_{0}\right)$ and the fluid depth $\left(z_{0}\right)$.

regularly with a certain period. This flow pattern resembles the phenomenon of so-called "wave number vacillation" appearing in the convection experiments in a rotating annulus. Since we could not get systematic data for this phenomenon, detailed description is beyond the scope of this paper. As for the hysterisis of the instability phenomena, it might be suspected to exist in the results. However, we could not detect certain evidences. In the following we focus our attention on the relationship between the observed wave numbers and external parameters.

b) Analysis of the dynamic process.

It is known that in the inviscid non-rotating system a jet with symmetrical velocity distribution is always unstable, if the velocity profile has an inflection point. Therefore, in discussions of dynamic instability we always consider some physical factors that stabilize the basic flow. The relevant factors in our experiment are considered as follows:

1) viscous dissipation in the interior region,

2) viscous dissipation in the Ekman layer,

3) beta-effect which is produced by the variation of the depth of the fluid layer,

4) divergence caused by the displacement of the free surface.

All the above factors except 3) show stabilizing effects for the flow regardless of the flow direction. The factor 3), however, shows different stability characteristics between westerly and easterly. In discussions associated with the generation of turbulence it is customary to deal only with the factor 1). In discussions associated with geophysical application the factor 3) is usually dealt with as the primary factor. Busse (1968) tries to explain the experimental results by Hide and Titman (1967) by the factor 2). Lipps (1963) gives a discussion about the effect of the factor 4).

It is expected that all these factors exist in our fluid system.

Besides,

5) circular geometry,

6) lateral boundary,

7) non-linear effect due to finite-amplitude disturbances.

It is desirable to assess the roles of these factors by making specially designed laboratory experiments or by forming a theory corresponding to our fluid system, but both are beyond the scope of this paper. We must content ourselves with getting some information for flow dynamics by comparing the observed data with relevant theories and experiments.

As shown in Lipps (1963, equation (2.5)) the effect of the factor 4) is negligible if the Froude number is very small compared with the nondimensional wave number and the beta term. In our experiment the Froude number defined by $\frac{0.25 \delta \Omega r_{0}}{\sqrt{g h}}\left(r_{0}\right.$ is the mean radius of the ring) is of the order of $10^{-2}$, which is very small compared with the magnitude of the wave number and the beta term $(0(1))$, so that the effect of the 

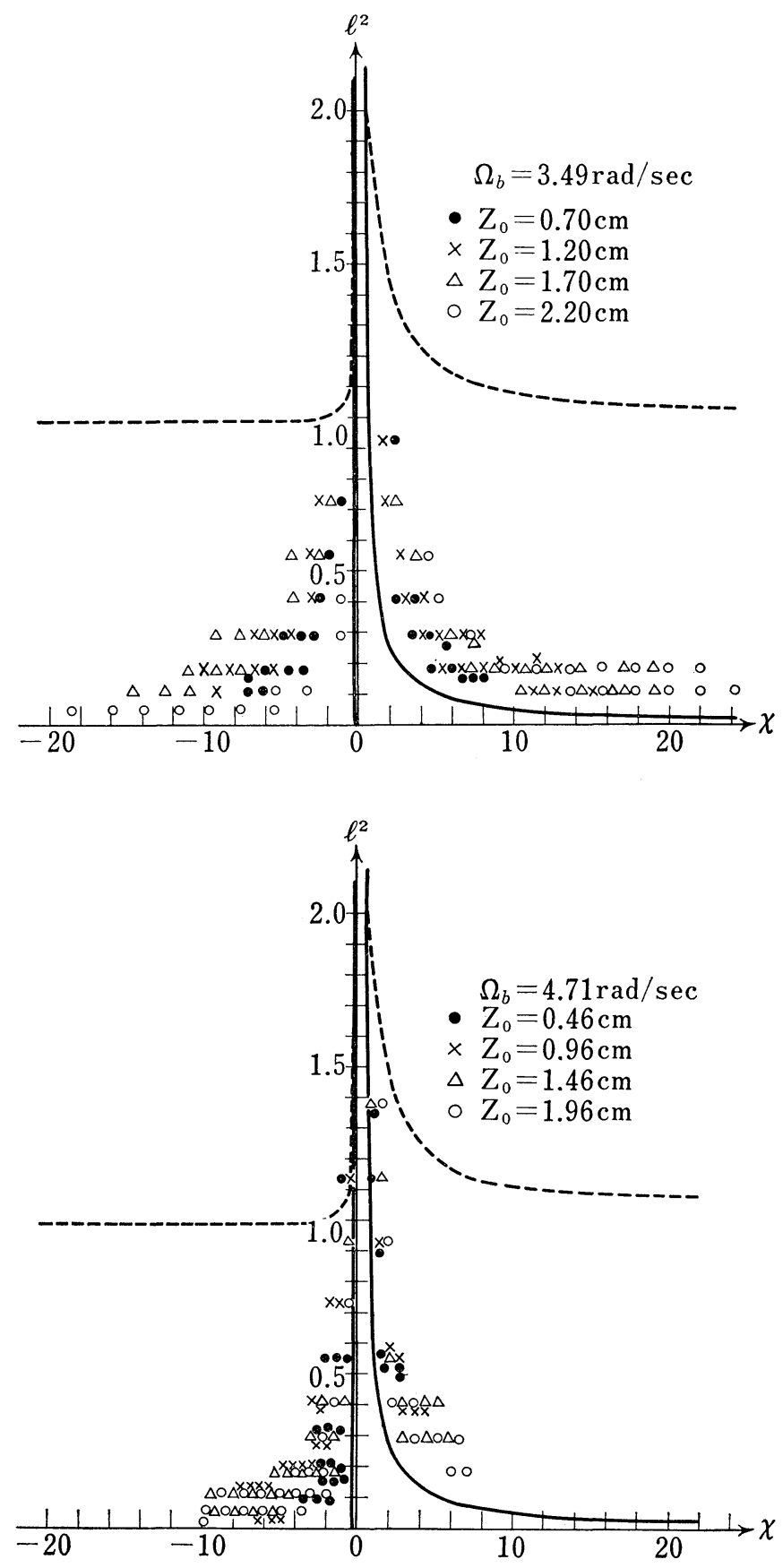

Fig. 10. Comparison of the theory and experiment for the wave numbers of disturbances. The observed data are plotted in Fig. 3 of Lipps (1962). The ordinate $l^{2}$ means the square of the non-dimensional wave number. The abscissa indicates the Rossby number which is defined by (6). 
factor 4) is considered to be negligible. The factors 1) and 2) have similar stabilizing effects. From the point of view of energetics, the ratio of the energy dissipation of the mean flow by those two factors is roughly estimated as

rate of viscous dissipation in the Ekman layer per unit time

rate of viscous dissipation in the vertical shear layer per unit time

$$
=\frac{\nu \times\left(v / E^{1 / 2} L\right)^{2} \times E^{1 / 2} L \times E^{1 / 4} L}{\nu \times\left(v / E^{1 / 4} L\right)^{2} \times L \times E^{1 / 4} L}=1
$$

where $v$ is the zonal flow velocity. Note that the above ratio is $0(1)$.

The effects of the factors 1) and 3) are studied by Tatsumi and Kakutani (1958) (referred to as $\mathrm{T}$ and $\mathrm{K}$ hereafter) and Lipps (1962), respectively. In both theories the velocity profile of the basic flow is assumed as $\operatorname{sech}^{2} b r$, where $b$ is a parameter which expresses the breadth of the jet. We put $b=1.5$ for our experimental situation as shown in Fig. 6. The non-dimensional parameter that expresses the intensity of the jet is the Reynolds numbers $R e$ defined by

$$
R e \equiv \frac{0.25 \cdot \delta \Omega \cdot r_{0}}{b \nu}
$$

in $T$ and $K$ and the Rossby number $\chi$ defined by

$$
\chi \equiv \frac{0.25 \cdot \delta \Omega \cdot r_{0} \cdot b^{2}}{3 \beta}
$$

in Lipps. In both theories the length is normalized by $b^{-1}$. The neutral curves obtained by both theories are shown by solid lines in Fig. 10 (Lipps) and Fig. 11 ( $\mathrm{T}$ and $\mathrm{K}$ ), respectively. In these figures $l$ expresses the non-dimensional wave number normalized by $b$. It should be noticed that the preferred wave number predicted by $\mathrm{T}$ and $\mathrm{K}(l \sim 0.2)$ is different from that predicted by Lipps $(l \sim 1.4)$. This difference is caused by the difference of the factor that stabilizes the basic flow.

We can compare the observed results with these theories by means of the following relations:

$$
l=\frac{k}{r_{0} \cdot b}
$$

where $k$ is the observed wave numbers as shown in Fig. 9,

$$
\beta=\frac{2 \Omega_{b}}{h} \cdot \frac{d h}{d r} \mid \text { at the ring }
$$
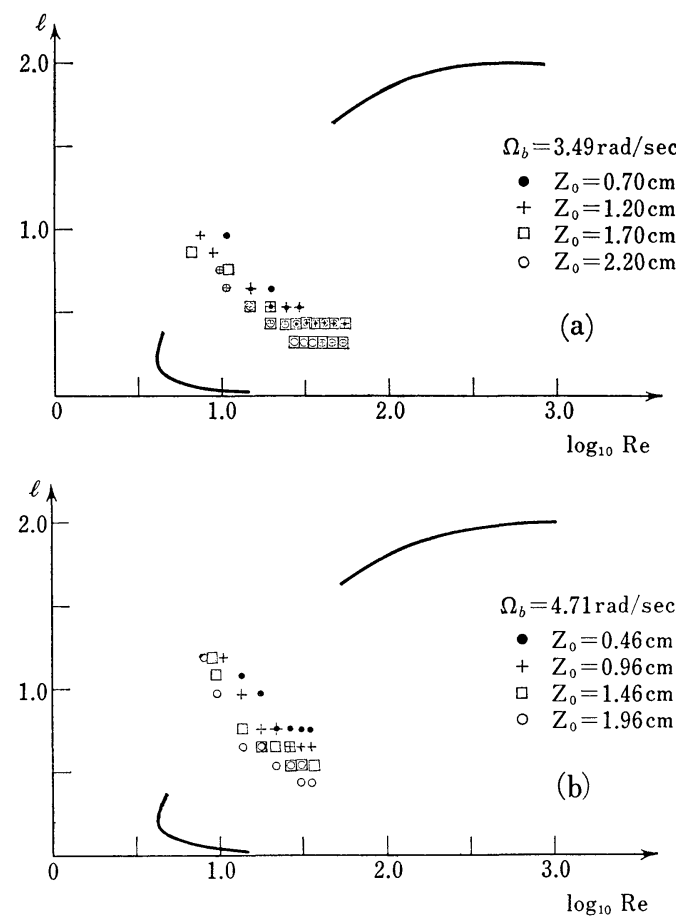

Fig. 11. Comparison of theory and experiment for the wave numbers of disturbances. The observed data are plotted in Fig. 1 of Tatsumi and Kakutani (1958). The ordinate indicates the non-dimensional wave number. The abscissa indicates the Reynolds number in logarithmic scale.

where $h$ is given by (1).

By plotting the experimental data in Figs. 10 and 11 we can estimate the role of the factors 1) and 3); if the observed data fit Lipps (Fig. 10) better than $T$ and $K$ (Fig. 11), it may be expected that the factor 3) plays a more important role in our fluid system than the factor 1) and vice versa. If the observed data do not fit either figure it may be expected that our fluid system is primarily controlled by the two factors or by others.

By plotting the observed data in Fig. 10 we notice following features:

i) All experimental data locate in the unstable regions predicted by the theory.

ii) We could not obtain the wave number at the marginal state in our experiment. But a rough extrapolation of the observed wave number to the marginal state suggests that the theory is not inconsistent with the 
observed result.

iii) The systematic dependence of the wave number on the fluid depth is more reduced than in Fig. 9, but does not disappear entirely.

iv) The experimental data well exhibit the following differences in stability property between the westerlies and easterlies;

a) The critical Rossby number for instability appears to be smaller in the easterly region than in the westerly region.

b) When the Rossby number $\chi$ is fixed, the observed wave numbers are smaller in the easterly region than in the westerly region.

By ploting the same data in Fig. 11 we notice the following features:

v) Some data are estimated to be located in the stable region predicted by the theory.

vi) Extrapolation of the observed wave numbers to the marginal state does not fit the linear preferred mode.

From the above results it may be concluded that the factor 3) is more important than the factor 1) as the controlling factor of the stabilizing property of the observed flow.

The feature iii) is partly explained by the relation (8). In our experimental situations, the increase of the fluid depth is accompanied with the decrease of the Ekman number as well as the decrease of $\beta$. Hence, the stabilizing effects by the factors 1) and 2) may be reduced. This will be the reason why the systematic dependence does not disappear. But the fact that the systematic dependence is more reduced in Fig. 10 than in Fig. 9 supports the importance of the $\beta$ effect in our experiment.

Difference in stability property between the westerlies and easterlies was discussed by Nitta and Yanai (1969). The feature iv) may be regarded as a rough verification of their theoretical result.

Since Lipps calculated only the neutral curve in the westerly region, we added the neutral curve in the easterly region and also the curves of the maximum growth rate (broken lines) in Fig. 10 by calculating them numerically. A striking result obtained in our experiment is that the observed wave numbers rapidly decrease in the finite amplitude region and do not fit the maximum growth rate curves. Hide and Titman (1967) also observed the decrease of the wave numbers in the finite amplitude region, although they did not compare their result with the linear preferred mode. According to the linear theory discussed by Busse (1968), the dissipation in the Ekman layer may reduce the growth rate of the disturbance, but does not alter the essential dynamical process. Therefore, we cannot expect that this is caused by the dissipation in the Ekman layer (the factor 2)). The effect of the factor 5) may be negligible, because the width of the jet is at most $10 \%$ of the radius of the ring. We cannot estimate the effects of the factors 6) and 7) within the scope of this paper. However, it is possible to explain this result qualitatively. Roughly speaking, the basic jet on which the finite amplitude disturbance is superposed can be considered as the new jet whose width is broader than that of the basic jet. Since the scale of the preferred unstable mode becomes large as the jet width becomes broad, it is expected that the realized scale of the disturbance increases more rapidly in the finite amplitude region than expected by the linear theory. These pictures, however, must be examined by more elaborate experimental and theoretical work.

\section{Acknowledgements}

This paper is based on the Master's Thesis presented by Toshio Yamagata to the Department of Geophysics of the University of Tokyo.

Primary research was performed at the Ocean Research Institute. The authors are grateful to Prof. K. Yoshida whose usual encouragements and suggestions were great help in this study. They are also indebted to Mr. A. Takeda for his support of this study. Thanks are extended to Mr. N. Misawa for his advice about the experimental apparatus and to Miss K. Yoshida for typing the manuscript. The authors also appreciate the careful reading and valuable comments on this paper by the reviewers.

\section{Appendix: Structure of the basic flow}

We consider a situation where

$$
L \ll r_{1}+2 a L=r_{2} \ll R \text {. }
$$

$2 a L$ is the width of the ring. Then, the fluid configuration near the ring region can be approximated by a straight channel model on a rotating system: The fluid with infinite extent is confined between two horizontal rigid planes whose vertical separation is $L$. We define a Cartesian coordinate system as shown in Fig. A-1. The ring is approximated by a strip region defined as $-a L \leq x \leq a L$ 


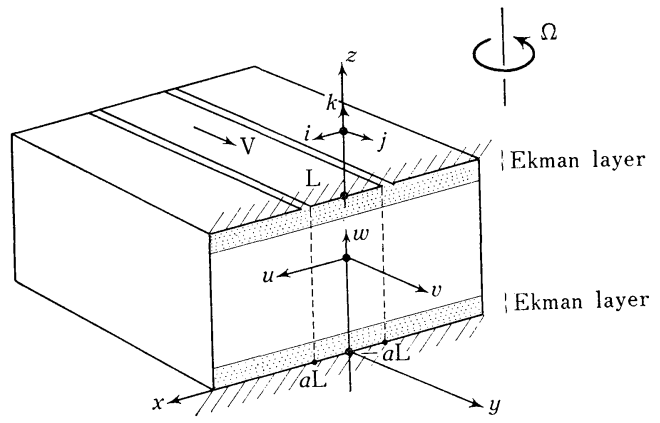

Fig. A-1. Flow configuration including coordinate system. $i, j, k$ are the unit vectors in the $x$-, $y$-, $z$-directions. $u, v, w$ are the flow velocities. $V$ is the velocity of the driving "ring".

and $z=L$, which moves in the $y$-direction with the velocity $V$ relative to the rotating system.

The governing equations for the fluid velocity $\boldsymbol{q}$ in the steady state are expressed by

$$
\begin{gathered}
\boldsymbol{q} \cdot \nabla \boldsymbol{q}+2 \Omega \boldsymbol{k} \times \boldsymbol{q}=-\frac{1}{\rho} \nabla p-g \boldsymbol{k}+\nu \nabla^{2} \boldsymbol{q} \\
\nabla \cdot \boldsymbol{q}=0
\end{gathered}
$$

with conventional notations. $\boldsymbol{k}$ is the unit vector in the $z$-direction.

By scaling $\boldsymbol{q}=\boldsymbol{q}^{*} V,(x, y, z)=\left(x^{*}, y^{*}, z^{*}\right) L, t=t^{*} \Omega^{-1}$, and $p=-\rho g z+p^{*} \rho V \Omega L,(\mathrm{~A}-1)$ and (A-2) are transformed into nondimensional forms:

$$
\begin{gathered}
\varepsilon \boldsymbol{q}^{*} \cdot \nabla^{*} \boldsymbol{q}^{*}+2 \boldsymbol{k} \times \boldsymbol{q}^{*}=-\nabla^{*} p^{*}+E \nabla^{* 2} \boldsymbol{q}^{*} \\
\nabla^{*} \cdot \boldsymbol{q}^{*}=0
\end{gathered}
$$

where $\epsilon\left(\equiv \frac{V}{\Omega L}\right)$ is the Rossby number and $E\left(\equiv \frac{\nu}{\Omega L^{2}}\right)$ is the Ekman number.

The boundary conditions are

$$
\left.\begin{array}{ll}
\boldsymbol{q}^{*}=0 & \text { at } z^{*}=0, \\
\boldsymbol{q}^{*}=V_{T} * \boldsymbol{j} & \text { as } z^{*}=1,
\end{array}\right\}
$$

where $V_{T}^{*}=1$ for $-a \leq x^{*} \leq a$ and $V_{T}^{*}=0$ for elsewhere.

The flow field is obtained by solving (A-3) and (A-4) under (A-5).

This problem is regarded as an example of vertical shear layers whose dynamics is discussed by Greenspan (1969, hereafter referred to as G.). In the following discussion we put $\epsilon=0$. This approximation is permitted as far as the deviation from the rigid rotation is small. It is known that the Ekman boundary layers with the thickness $(\nu / \Omega)^{1 / 2}$ are formed near the top and bottom boundaries and the dynamics of the inner region (outside of the Ekman layer) can be expressed with simplified equations of (A-3) and (A-4):

$$
\left.\begin{array}{rl}
-2 v & =-\frac{\partial p}{\partial x} \\
2 u & =\quad E \frac{\partial^{2} v}{\partial x^{2}} \\
0 & =-\frac{\partial p}{\partial z}+E \frac{\partial^{2} w}{\partial x^{2}} \\
0 & =\frac{\partial u}{\partial x}+\frac{\partial w}{\partial z}
\end{array}\right\}
$$

where $q=(u, v, w)$ and we omit the asterisk in the non-dimensional quantities. (A-6) corresponds to the equation system for the vertical shear layer of thickness $E^{1 / 3}$ (see p. 99 of G.).

Since (A-6) is only valid in the inner region, we cannot use (A-5) as the boundary condition for (A-6). The appropriate boundary condition for (A-6) is given through the vertical velocity induced by Ekman convergence:

$$
\left.\begin{array}{l}
w=\frac{1}{2} \cdot E^{1 / 2} \frac{\partial v}{\partial x} \quad \text { at } z=0 \\
w=-\frac{1}{2} \cdot E^{1 / 2} \frac{\partial}{\partial x}\left(v-V_{T}\right) \text { at } z=1
\end{array}\right\}
$$

(A-7) is derived from (2.17.3) in G. by neglecting the contribution of $u$ which is negligible compared with that of $v$. Notice that the driving of the flow by $V_{T}$ is effective only at $x= \pm a$. The singular characteristic of $w$ at these points is due to neglecting the horizontal diffusion in the Ekman layer in deriving (2.17.3) in G.. In the following calculation we expect that the influence by this singular characteristic of $w$ is confined near $x= \pm a$ and does not violate the whole flow fields. This will be checked by comparing the result of calculation with our experiments. The nondimensional thickness of the Ekman layer is $E^{1 / 2}$. Therefore, strictly speaking, the boundary condition (A-7) should be given at $z=E^{1 / 2}$ and $z=1-E^{1 / 2}$. Since $E^{1 / 2}$ is very small in our problem, we neglect the thickness of the Ekman layer in (A-7).

By introducing a stream function $\phi$ which is defined by

$$
u=\frac{\partial \psi}{\partial z}, \quad w=-\frac{\partial \psi}{\partial x}
$$


(A-6) is reduced to

$$
\left.\begin{array}{l}
E \frac{\partial^{2} v}{\partial x^{2}}-2 \frac{\partial \psi}{\partial v}=0 \\
E \frac{\partial^{4} \psi}{\partial x^{4}}+2 \frac{\partial v}{\partial z}=0
\end{array}\right\}
$$

(A-9) (A-14) shows the positions near the origin and (A-15) shows those of the main term of the denominator in (A-12). Under these approximations $v$ is expressed by

$$
v(x, z) \fallingdotseq v_{1}(x, z)+v_{2}(x, z)
$$

(A-10) where

$$
\left.\begin{array}{ll}
\psi+\frac{1}{2} E^{1 / 2} v=0 & \text { at } z=0 \\
\psi-\frac{1}{2} E^{1 / 2}\left(v-V_{T}\right)=0 & \text { at } z=1
\end{array}\right\}
$$

Thus, our problem is reduced to solving (A-9) under the boundary condition (A-10).

The solution can be obtained by means of the Fourier transform method. If we define $\bar{v}$ and $\bar{\psi}$ as

$$
\left.\begin{array}{l}
\bar{v}(\xi, z)=\frac{1}{\sqrt{2 \pi}} \int_{-\infty}^{\infty} v(x, z) e^{-i x \xi} d x \\
\bar{\psi}(\xi, z)=\frac{1}{\sqrt{2 \pi}} \int_{-\infty}^{\infty} \psi(x, z) e^{-i x \xi} d x
\end{array}\right\}
$$

$\bar{v}$ and $\bar{\psi}$ which satisfy the boundary condition (A-10) are expressed by

$$
\left.\begin{array}{c}
\bar{v}=\frac{\xi \bar{V}_{T}\left(\xi \sinh \frac{E}{2} \xi^{3} z+\frac{2}{E^{1 / 2}} \cosh \frac{E}{2} \xi^{3} z\right)}{\left(\xi^{2}+\frac{4}{E}\right) \sinh \frac{E}{2} \xi^{3}+\frac{4}{E^{1 / 2}} \xi \cdot \cosh \frac{E}{2} \xi^{3}} \\
\bar{\psi}=\frac{-\bar{V}_{T}\left(\xi \cosh \frac{E}{2} \xi^{3}+\frac{4}{E^{1 / 2}} \sinh \frac{E}{2} \xi^{3} z\right)}{\left(\xi^{2}+\frac{4}{E}\right) \sinh \frac{E}{2} \xi^{3}+\frac{4}{E^{1 / 2}} \cdot \xi \cdot \cosh \frac{E}{2} \xi^{3}}
\end{array}\right\}
$$

where $\bar{V}_{T}$ is the Fourier transform of $V_{T}$ whose explicit form is given by

$$
\begin{aligned}
\bar{V}_{T} & =\frac{1}{\sqrt{2 \pi}} \int_{-\infty}^{\infty} V_{T} \cdot e^{-i \xi x} d x \\
& =\frac{1}{\sqrt{2 \pi}} \int_{-a}^{a} e^{-i \xi x} d x=-\frac{i}{\sqrt{2 \pi}} \cdot \frac{1}{\xi}\left(e^{i \xi a}-e^{-i \xi a}\right)
\end{aligned}
$$

The explicit form of $v(x, z)$ and $\phi(x, z)$ is obtained by substituting (A-12) into (A-11) and performing the integration. It is not easy to obtain the exact integral, but the approximate value can be found by means of residue calculations. The singularities of the integrand are located at the approximate positions given by the roots of

$$
\xi\left(\xi^{2}+\frac{2}{E^{1 / 2}}\right)=0
$$

$$
v_{1}(x, z)=\frac{1}{\sqrt{2 \pi}} \cdot \frac{2}{E^{1 / 2}} \cdot \int_{-\infty}^{\infty} \frac{\xi \bar{V}_{T}}{2 \xi\left(\xi^{2}+\frac{2}{E^{1 / 2}}\right)} \cdot e^{i x \xi} \cdot d \xi
$$

$$
v_{2}(x, z)=\frac{1}{\sqrt{2 \pi}} \cdot \frac{E^{1 / 2}}{2} \cdot \int_{-\infty}^{\infty} \frac{\xi \bar{V}_{T} \cdot \cosh \frac{E}{2} \xi^{3} z}{\sinh \frac{E}{2} \xi^{3}} \cdot e^{2 x \xi} \cdot d \xi
$$

and (A-4) is expressed by

$$
\phi(x, z) \fallingdotseq \phi_{1}(x, z)+\phi_{2}(x, z)
$$

where

$$
\begin{aligned}
& \phi_{1}(x, z)=-\frac{1}{\sqrt{2 \pi}} \int_{-\infty}^{\infty} \frac{\bar{V}_{T} \cdot \xi \cdot\left(1+E^{1 / 2} \xi^{2} z\right)}{2 \cdot \xi \cdot\left(\xi^{2}+\frac{2}{E^{1 / 2}}\right)} \cdot e^{i x \xi} d \xi \\
& (\mathrm{A}-20) \\
& \psi_{2}(x, z)=-\frac{1}{\sqrt{2 \pi}} \cdot \frac{E^{1 / 2}}{2} \int_{-\infty}^{\infty} \frac{V_{T} \cdot \sinh \frac{E}{2} \xi^{3} z}{\sinh \frac{E}{2} \xi^{3}} \cdot e^{i x \xi} d \xi
\end{aligned}
$$

The residue calculation yields the following explicit forms for the flow field:

(i) for $x<-a$

$$
\begin{aligned}
& v_{1}=\frac{1}{2} e^{\left(\frac{4}{E}\right)^{1 / 4} x} \sinh \left(\frac{4}{E}\right)^{1 / 4} a \\
& v_{1}=\frac{2}{3} E^{1 / 6} \sum_{n=1}^{\infty} \frac{(-1)^{n} \cdot e^{\gamma_{n} x} \cos n \pi z}{(2 n \pi)^{2 / 3}} \cdot \sinh \gamma_{n}(x+a) \\
& \text { where } \gamma_{n} \equiv\left(\frac{2 n \pi}{E}\right)^{1 / 3} \\
& \phi_{1}=-\frac{E^{1 / 2}}{2}(1-2 z) v_{1} \\
& \phi_{2}=\frac{E^{1 / 2}}{3 \pi} \sum_{n=1}^{\infty} \frac{(-1)^{n^{n} \sin n \pi z}}{n} \cdot e^{\gamma_{n} x} \cdot \sinh \gamma_{n} a
\end{aligned}
$$


(ii) for $-a<x<a$

$$
\begin{aligned}
v_{1} & =\frac{1}{2}\left(1-e^{-\left(\frac{4}{E}\right)^{1 / 4} a} \cosh \left(\frac{4}{E}\right)^{1 / 4} x\right) \\
v_{2} & =\frac{2}{3} E^{1 / 6} \sum_{n=1}^{\infty} \frac{(-1)^{n} \cos n \pi z}{(2 n \pi)^{2 / 3}}\left\{e^{-\frac{1}{2}-\gamma_{n}(x+a)}\right. \\
& \left.\times \cos \left[\gamma_{n} \frac{\sqrt{3}}{2}(x+a)-\frac{\pi}{3}\right]-\frac{1}{2} e^{\gamma_{n}(x-a)}\right\} \\
\phi_{1} & =-\frac{E^{1 / 2}}{2}(1-2 z) v_{1} \\
\phi_{2} & =-\frac{E^{1 / 2}}{6 \pi} \sum_{n=1}^{\infty} \frac{(-1)^{n} \sin n \pi z}{n}\left\{2 e^{-1 / 2 \gamma_{n}(x+a)}\right. \\
& \left.\times \cos \gamma_{n} \frac{\sqrt{3}}{2}(x+a)+e^{\gamma_{n}(x-a)}\right\}
\end{aligned}
$$

(iii) for $x>a$

$$
\begin{aligned}
& v_{1}=\frac{1}{2} e^{-\left(\frac{4}{E}\right)^{1 / 4} x} \sinh \left(\frac{4}{E}\right)^{1 / 4} a \\
& v_{2}=\frac{2}{3} E^{1 / 6} \sum_{n=1}^{\infty} \frac{(-1)^{n} \cdot \cos n \pi z}{(2 n \pi)^{2 / 3}}\left\{e^{-1 / 2 \gamma_{n}(x+a)}\right. \\
& \times \cos \left(\gamma_{n} \frac{\sqrt{3}}{2}(x+a)-\frac{\pi}{3}\right)-e^{-1 / 2 \gamma_{n}(x-a)} \\
& \left.\times \cos \left(\gamma_{n} \frac{\sqrt{3}}{2}(x-a)-\frac{\pi}{3}\right)\right\} \\
& \phi_{1}=-\frac{E^{1 / 2}}{2}(1-2 z) v_{1} \\
& \phi_{2}=-\frac{E^{1 / 2}}{3 \pi} \sum_{n=1}^{\infty} \frac{(-1)^{n} \sin n \pi z}{n}\left\{e^{-1 / 2 \gamma_{n}(x+a)}\right. \\
& \times \cos \gamma_{n} \frac{\sqrt{3}}{2}(x+a)-e^{-1 / 2 \gamma_{n}(x-a)} \\
& \left.\times \cos \gamma_{n} \frac{\sqrt{3}}{2}(x-a)\right\}
\end{aligned}
$$

Considering $E \ll 1$, it is seen from these formulae that the main feature of the basic flow is determined by (A-17) and the amplitude of the meridional circulation is $E^{1 / 2}$ compared with the unit zonal flow. Besides, $v_{1}$ does not depend on $z$, which means that the zonal flow is nearly two-dimensional in our fluid configuration.

The amplitude of the zonal flow is approximately given by

$$
v_{\max } \fallingdotseq \frac{1}{2}\left(1-e^{-\left(\frac{4}{E}\right)^{1 / 4} a}\right)
$$

from (A-26). Notice that the amplitude varies from $1 / 2$ to 0 depending upon the width of the ring.

The explict forms of (A-18) and (A-21) are expressed in the form of series expansion. The solutions converge at the first several terms except for the region near the driving points; $(x, z)=$ $( \pm a, 1)$. Fig. A-2 shows the tendency of the

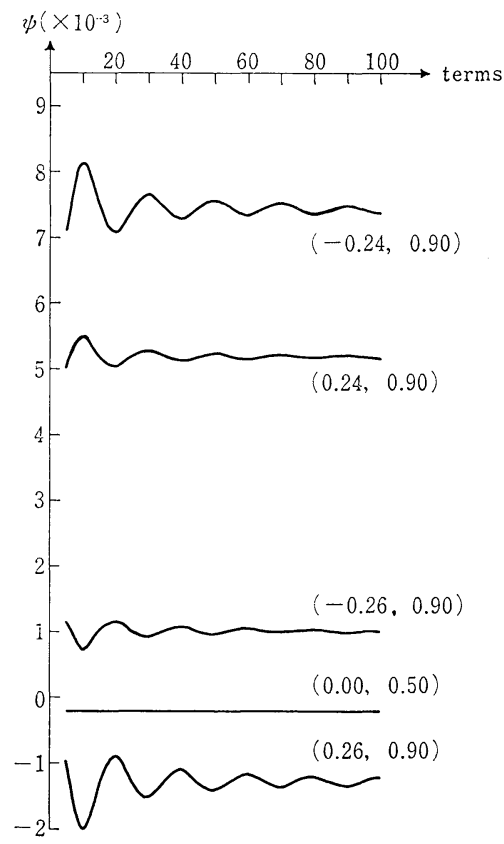

Fig. A-2. Tendency of the convergence for the stream function $\psi$ at various points. The locations are represented by $(x, z), \psi$ is shown as a function of the number of the calculated terms of the infinite series appeared in $\psi_{2}$.

convergence for $\psi$ with increase of the number of the calculated terms.

A typical horizontal profile of the zonal velocity (for $E=10^{-3}, a=0.25$ ) is shown in Fig. A-3. The velocities were calculated by truncating the infinite series in (A-18) and (A-21) at the 50th term except for the profile at $z=1.0$, which was obtained by truncating them at the 100th term. The calculated zonal velocity shows sharp jet-like flows near the driving points, but they are confined in the thin layer next to the upper boundary layer. It can 


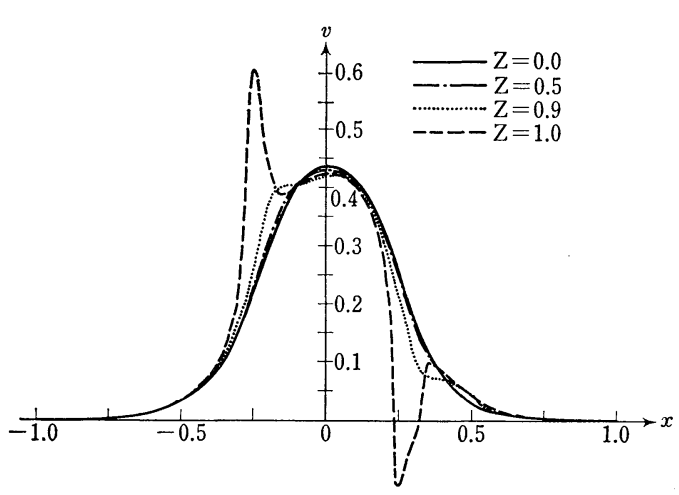

Fig. A-3. Typical profiles of $v$ at various depths for $E=10^{-3}, a=0.25$.

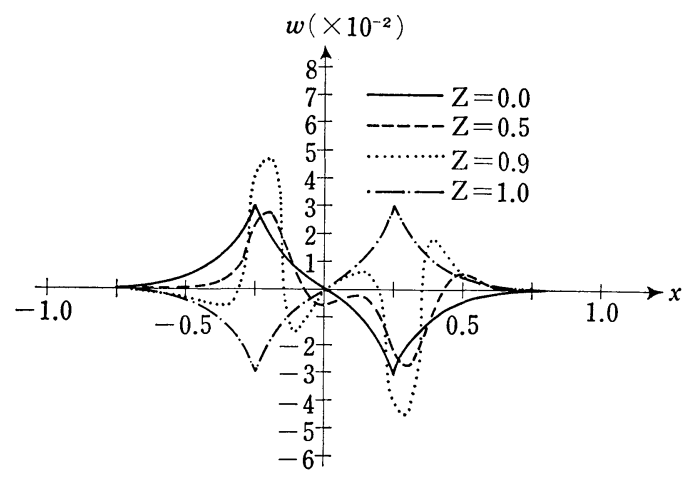

Fig. A-4. Typical profiles of $w$ at various depths for $E=10^{-3}, a=0.25$.

be regarded that the flow is almost twodimensional below $z=0.9$. The inflection points are located near $x= \pm a$.

Fig A-4 shows horizonal profiles of the vertical velocity corresponding to the zonal velocity shown in Fig. A-3. Looking downstream, we find a typical downward flow on the righthand side of the axis of the zonal flow. The fluid sent from the upper boundary layer into the interior region by Ekman convergence is transported to the lower boundary layer by this downward flow. On the other hand, at the left hand side of the zonal flow the fluid is transported from the lower to the upper boundary layer to complete the meridional circulation. The above circulation has a tendency to make a single-cell structure. On the other hand there exists another meridional circulation which is secondarily produced by the zonal flow. This tends to make a double-cell structure. We find the composite patterns of a single-cell and a double-cell structures in Fig. A-4. The amplitude is of the order of $E^{1 / 2}$ compared with the unit amplitude of the zonal flow.

The calculated $w$ expresses the essential characteristic of the meridional circulation, but the accuracy is doubtful for the detailed structure, because the solutions show the following unrealistic features:

1) $\phi$ is not continuous at $z= \pm a$ except $z=0$ and 1 , so that $w$ does not satisfy the upper boundary condition and the infinite values appear in the interior region at $x= \pm a$.

2) Horizontal gradient of $w$ is not continuous at $x= \pm a$ and $w$ at $z=0$ is unrealistic, as shown in Fig. A-4. Approximations in the boundary condition (A-7) and for the positions of the singularities in the residue calculation of (A-12) may be responsible for these results.

\section{References}

Baker, D.J., 1966: A technique for the precise measurement of small fluid velocities. J. Fluid Mech., 26, 573-575.

Busse, F.H., 1968: Shear flow instabilities in rotating systems. J. Fluid Mech., 33, 577-589.

Greenspan, H.P., 1969: The theory of rotating fluids. Cambridge University Press, 92, 99.

Hide, R. and Titman, C.W., 1967: Detached shear layers in a rotating fluid. J. Fluid Mech., 29, 3960.

Kimura, R., Tsu, H. and Yagihashi, A., 1971: Convective patterns in a plane Coutte flow. J. Meteor. Soc. Japan, 49, 249-260.

Kuo, H., 1949: Dynamic instability of two-dimensional nondivergent flow in a barotropic atmosphere. J. Meteor., 6, 105-122.

Lipps, F.B., 1962: The barotropic stability of the mean winds in the atmosphere. J. Fluid Mech., 12, 397407.

, 1963: Stability of jets in a divergent barotropic fluid. J. Atm. Sci. 20, 120-129.

- 1965:The The stability of an asymmetric zonal current in the atmosphere. J. Fluid Mech, 21, 225-239.

Sato, H., 1959: The stability and transition of a twodimensional jet. J. Fluid Mech., 7, 53-80.

Schlichting, H., 1968: Boundary-layer theory. McGrawHill Book Company, 93.

Schraub, F.A. et al., 1965: Use of hydrogen bubbles for quantitative determination of time-dependent velocity fields in lowspeed water flows. J. Basic Engineering, 87, 429-444.

Tatsumi, T. and Kakutani, T. 1958: The stability of a two-dimensional laminar jet. J. Fluid Mech., 4, 261-275. 
Yamasaki, M. and Wada, M., 1972: Barotropic Yanai, M. and Nitta, T., 1968: Finite difference instability of an easterly zonal current. J. Meteor. Soc. Japan, 50, 101-121. approximations for the barotropic instability problem. J. Meteor. Soc. Japan, 46, 389-403.

\title{
回転系における準二次元的なジェットのカ学不安定（順圧不安定）の実験
}

\author{
山 形 俊 男 \\ 東京大学理学部地球物理学教室 (現在海洋研究所) \\ 木 村 竜治 \\ 東京大学海洋研究所
}

回転系の力学不安定現象を室内実験により調べた，基本流は剛体回転をしている流体の自由表面を平板でこすって 作った．基本流の構造は線型論をもちいて議論した。ベータ効果は流体の深さの変化に伴う渦系の伸縮を利用して表 現した.

ロスビー数が臨界值を越えると，基本流は不安定になり，一定の波数の擾乱が現われた，波数の測定結果と線型論 の結果とを比較して次の結果を得た.

(i) 臨界状態付近で測定された波数は, 内部粘性を基本流の安定化要素と考学る理論による予想よりも, ベータ効 果を安定化要素と考光る理論による予想の方に近かった。

（ii）水深が回転中心から遠ざかるに従って深くなる系では，偏西風に対応する基本流の方が，偏東風に対応する基 本流よりる，ょり安定であった.

(iii) 臨界值を越えてロスビー数をさらに大きくすると, 測定された波数は, 線型論で予想される波数より急激に小 さくなった. 